Research Article

\title{
Application of Green Synthesized Metal Nanoparticles in the Photocatalytic Degradation of Dyes and Its Mathematical Modelling Using the Caputo-Fabrizio Fractional Derivative without the Singular Kernel
}

\author{
S. Dave $\mathbb{D}^{1},{ }^{1}$ A. M. Khan $\mathbb{D}^{2},{ }^{2}$ S. D. Purohit $\mathbb{D}^{3},{ }^{3}$ and D. L. Suthar $\mathbb{D}^{4}$ \\ ${ }^{1}$ Department of Chemistry, Jodhpur Institute of Engineering \& Technology, Jodhpur, India \\ ${ }^{2}$ Department of Mathematics, Jodhpur Institute of Engineering \& Technology, Jodhpur, India \\ ${ }^{3}$ Department of Mathematics, HEAS, Rajasthan Technical University, Kota, India \\ ${ }^{4}$ Department of Mathematics, Wollo University, Dessie Campus, Wollo Dessie, Amhara, Ethiopia
}

Correspondence should be addressed to D. L. Suthar; dlsuthar@gmail.com

Received 23 March 2021; Accepted 25 May 2021; Published 4 June 2021

Academic Editor: Ahmet Ocak Akdemir

Copyright ( 92021 S. Dave et al. This is an open access article distributed under the Creative Commons Attribution License, which permits unrestricted use, distribution, and reproduction in any medium, provided the original work is properly cited.

Textile dyes are untreated discharge into the environment which results in a significant increase in water pollution levels worldwide. Due to the continuous addition of toxic organic dyes, a necessary strategic model is required for the complete degradation of dyes in textile effluent. This paper considers the possibility of biological synthesis of silver and iron nanoparticles and their use in photocatalytic degradation. The immediate change of silver nitrate solution occurring from colorless to brown is observed after the addition of the aqueous leaf extract, indicating the successive reduction of Ag+ ions to the Ag nanoparticles. These formed Ag nanoparticles were subjected to examine the photocatalytic activity under the solar radiation for the degradation of methyl orange. Green synthesized Ag nanoparticles were found to successfully degrade methyl orange up to $95 \%$ between 70 hours than the initial exposure time. The absorbance of methyl orange was measured at $465 \mathrm{~nm}$. The present paper is focused on fractional mathematical modelling of dye degradation in textile effluents using the Caputo-Fabrizio fractional derivative without the singular kernel. The iterative Laplace transform method is employed to obtain an analytic solution for the absorption transport equation. The obtained experimental results showing significant removal of dyes from textile wastewater are compared using modelling results. The innovative approach is in outstanding agreement with the findings of the experiment. The mathematical modelling for the dye removal process helps to design suitable environmental management studies to reduce the adverse effect caused by toxic wastewater. Model validation has been shown by comparing analytical simulated solutions with experimental results for photocatalytic degradation using silver and iron nanoparticles as eco-friendly and low-cost agents.

\section{Introduction}

Dyes are the most important type of synthetic organic materials utilized in various industries such as textiles, food, and pharmaceuticals. The basic strategy for the remediation of these dye compounds from manufacturing effluents has been accompanied by the use of chemical reagents, physical aspects, and biological processes. However, these methods are laborious and inefficient and have issues with disposal as well.
Recently, in [1], photocatalytic activity by metal nanoparticles sought significant attention due to the fact that it has the characteristic properties of degrading organic compounds under solar light illumination in the case of metal catalysts. Compared to traditional approaches, this process is low cost and does not produce toxic goods. Nanotechnology allows the development of nanoparticles with regulated size, design, and variance of materials at the nanometer scale length, with the aim of using them to enhance human health. Metal 
nanoparticles, among all nanoparticles, have a broad variety of applications in areas such as bioimaging, sensor growth, and data processing and novel applications in the biomedical research sector. The late application of metallic silver and silver nanoparticles as antimicrobial operators in various products started, for example, powder and paint, animal feed, covering of the catheter tube, wound patch dressing materials, and water purifying treatments [2], with a negligible danger of toxification in human beings. The green methodology of nanoparticles prepared from natural substances is gaining incredible popularity because it is more environmentally friendly, less harmful, and less time consuming; at present, plant materials are utilized for nanoparticles' formation because they are more perfect than the microorganism-mediated nanoparticles' procedure since they are difficult to handle.

Plant extract-based synthesis of nanoparticles is having tremendous success due to its compatibility, environmentallyfriendly, and least time consuming properties [3-5]. In a recent study, silver nanoparticles were effectively fabricated using the Cordia dichotoma (common name: gonda) leaf extract, and the silver and iron nanoparticles synthesized were used in the degradation of dyes. A flowering plant Cordia dichotoma is species from the family of borage, and it is boraginaceous which is native to the regions of western Melanesia, northern Australia, and Indomalayan realm. Common vernacular names include Indian cherry, bird lime tree, pink pearl, glue berry, anonang, cumming cordia, snotty gobbles, fragrant manjack, and lasoda (gunda), respectively. Cordia dichotoma is a deciduous tree with a short bole and a spreading crown that grows to be small to intermediate in height. The stem bark is greyish brown in color and can be smooth or wrinkled over its base. The flowers are short stalked, whitish, and open only at night. The fruit is smooth, green-yellow, or pink-yellow globose that becomes black after ripening, and the pulp becomes viscid. Figure 1 depicts plants and their leaves found in tropical and subtropical regions. It can be found in a variety of forests, from the dry deciduous forests of Rajasthan to the wet deciduous forests of the Western Ghats and the coastal forests of Myanmar. Fabricated silver nanoparticles under exposure to sunlight have been exposed to dye degradation operation. Though a lot of work has been done to measure the performance of many adsorbents for dye degradation from industries, yet very little work has been done to model the dye degradation process to evaluate the effect of various parameters on the dye degradation process. In [6], modelling enables the future prediction and indicates the importance of various factors in the real system. The numerical iterative Laplace transform method is employed to simulate the degradation process of dyes from wastewater. The findings achieved by the proposed model could help to refine the wastewater management strategy.

\section{Experimental}

In order to assess the validity of the numerical modelling for the analysis of wastewater dye degradation, the simulated findings are compared with the results of the experimental studies. In the laboratory test, the following materials and methods were followed.

2.1. Preparation of the Plant Extract. Leaves of Cordia dichotoma (common name: gonda) were collected from the JIET campus. $10 \mathrm{~g}$ of fresh leaves were sliced into thin pieces and washed vigorously with double-distilled water. The leaves' content was added with $100 \mathrm{~mL}$ of double-distilled water and kept for boiling at $60^{\circ} \mathrm{C}$ for $10 \mathrm{~min}$. Then, the filtrate was obtained by passing the boiled mixture through Whatman No. 1 filter paper and kept in a clean container at $4^{\circ} \mathrm{C}$ for further nanoparticle synthesis process.

2.2. Biosynthesis of Silver Nanoparticles. About 1 millimolar silver nitrate aqueous salt solution was prepared in doubledistilled water that was procured from Sigma-Aldrich grade salt. Appropriately, $5 \mathrm{~mL}$ of the freshly prepared leaf extract was mixed with $45 \mathrm{~mL}$ of aqueous silver nitrate salt solution. For the method of reducing the silver ion to silver nanoparticles, the mixture was held for incubation at room temperature. The formation of silver NPs was identified visibly as the solution turns colorless to brown and later identified using the UV-vis spectrum analysis. The variation in $\mathrm{pH}$ of the leaf extract was altered to examine the effect of the production of silver nanoparticles (Figure 2). The UV-vis spectrophotometer measured the formation of silver nanoparticles at a wide range of wavelengths. The method followed is described in the earlier work published by Dave [7].

2.3. Biosynthesis of Iron Nanoparticles. Aqueous salt solution of ferrous sulphate was formulated using double-distilled water at a concentration of $1 \mathrm{mM}$ of $5 \mathrm{~mL}$ of the newly developed leaf extract which was applied to $45 \mathrm{~mL}$ of aqueous salt solution of ferrous sulphate and stored at room temperature for the reduction of Fe nanoparticles (Figure 3).

2.4. Characterization of Biosynthesized Silver Nanoparticles Using UV-Vis Spectroscopy. The purified silver nanoparticles were obtained using repeated centrifugation method at $7000 \mathrm{rpm}$ for $15 \mathrm{~min}$ followed by drying at $100^{\circ} \mathrm{C}$. The successive reduction of silver nitrate into silver NPs was subjected to the double-beam UV-vis spectrophotometer for measuring the spectrum at a differential wavelength from $360 \mathrm{~nm}$ to $700 \mathrm{~nm}$, respectively.

\subsection{Photocatalytic Degradation of Dye}

2.5.1. Using Silver Nanoparticles. From the biosynthesized silver nanoparticles, $5 \mathrm{mg}$ Ag NPs was added to the test flask containing $50 \mathrm{~mL}$ of methyl orange dye solution. The control test bottle was also preserved without the inclusion of silver nanoparticles. Until exposure to sunlight irradiation, the reaction suspension was thoroughly combined with magnetic stirring for $30 \mathrm{~min}$ to clearly align the operating test solution. Subsequently, the dispersed solution was put under sunlight and monitored for color change significant to the 

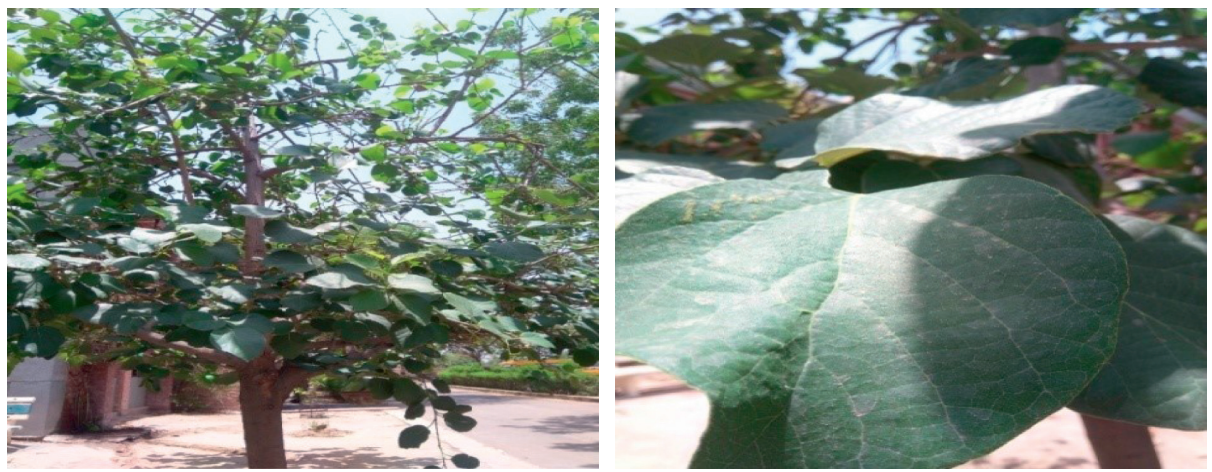

Figure 1: The plant and its leaf.

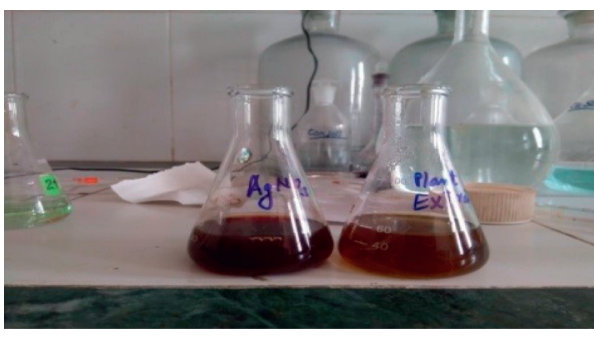

Figure 2: Synthesis of silver nanoparticles.

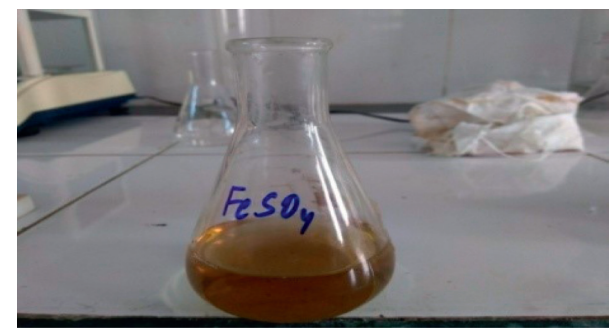

FIGURE 3: Synthesis of iron nanoparticles.

production of Ag NPs. At specific time intervals, aliquots of 2-3 mL from the suspension were screened and used to analyze the photocatalytic degradation activities of the dye using the UV-vis spectrophotometer at various frequencies. The degrading (Figure 4) concentration of the dye during the degradation operation was determined by observing the suspension solution value at $660 \mathrm{~nm}$.

2.5.2. Using Iron Nanoparticles. For reactions similar to Fenton oxidation, iron nanoparticles were prepared using ferrous sulphate as a precursor, and $1 \mathrm{ml}$ of colloidal iron nanoparticles along with $1 \mathrm{ml}$ of $3 \% \mathrm{H}_{2} \mathrm{O}_{2}$ was added to $9 \mathrm{ml}$ of $50 \mathrm{ppm}$ methyl orange in a test tube. Five replicates were prepared for each sample. A new blank (control) was also included in each round of dye degradation, containing the same volume of the dye and $\mathrm{H}_{2} \mathrm{O}_{2}$ but without colloidal water replacing the colloidal nanoparticles. The concentration was measured using a UV-vis spectrophotometer. The percentage of dye degradation was calculated using the preceding formula where the initial concentration of dye

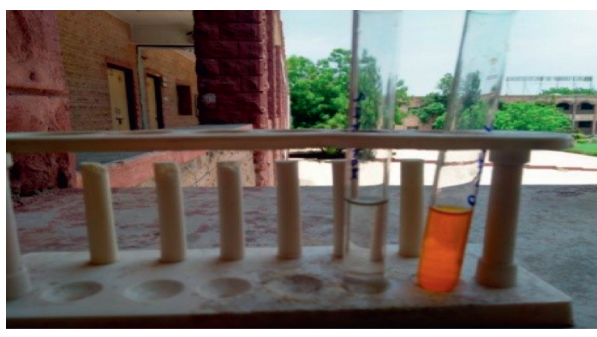

Figure 4: Dye degradation using silver nanoparticles.

solution and concentration of dye solution are present after photocatalytic degradation (Figure 5).

\section{Mathematical Modelling Using the Caputo-Fabrizio Fractional Derivative without the Singular Kernel}

In this article, we based on a fractional-order mathematical model to investigate the transport of relevant textile industry effluents by using the Caputo-Fabrizio fractional derivative without the singular kernel (see also [8]). The concentration of analytical solution is obtained by the iterative Laplace transform technique, and the concentration is plotted for different input parameters. For more modern fractionalorder mathematical model developments, the reader can refer to [9-16].

The transport equation due to Doulati Ardejani et al. [17] for the absorption process is given as

$$
R \frac{\partial C}{\mathrm{~d} t}=-K S \rho_{d}
$$

where

$C$ : the concentration of solution

$S$ : the quantity of absorbed mass on the surface

$R$ : the retardation factor

$K$ : the delay constant

$\rho_{d}$ : the bulk density of the medium

The relationship between $C$ and $S$ due to the Langmuir isotherm [18] is given as 


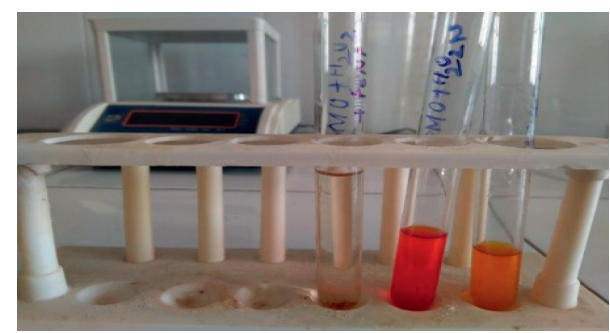

Figure 5: Dye degradation using iron nanoparticles.

$$
S=\frac{Q_{0} K_{L} C}{1+K_{L} C}
$$

where

$Q_{0}$ : the maximum absorption capacity

$K_{L}$ : the Langmuir constant

Using (1) and (2), we have

$$
R \frac{\partial C}{\mathrm{~d} t}=-\frac{K K_{L} Q_{0} \rho_{d} C}{1+K_{L} C},
$$

with $C(0)=C_{0}$.

Let $L(a, b)=\left\{f: f \in L^{2}(a, b)\right.$ and $\left.f^{\prime} \in L^{2}(a, b)\right\}$, where $L^{2}(a, b)$ is the space of square-integrable functions on interval $(a, b)$. Furthermore, $H(0, b)=\left\{f: f \in L^{2}(0, b)\right.$ and $\left.f^{\prime} \in L^{2}(0, b)\right\}$, with $b>0$.

Definition 1. Let $0<\alpha<1$; the fractional Caputo-Fabrizio $[19,20]$ derivative of order $\alpha$ for a function $f(t) \in H(0, b)$ with $b>0$ is given by

$$
{ }^{\mathrm{CF}} \mathfrak{D}_{t}^{\alpha} f(t)=\frac{(2-\alpha) M(\alpha)}{2(1-\alpha)} \int_{0}^{t} f^{\prime}(x) \exp \left[\frac{-\alpha}{1-\alpha}(t-x)\right] \mathrm{d} x,
$$

$t \geq 0$, where $M(\alpha)$ is the normalization function.

Definition 2. Let $0<\alpha<1$; the fractional integral of order $\alpha$ for a function $f$ is defined as

$$
\begin{aligned}
\mathrm{CF}^{\alpha} f(t)= & \frac{2(1-\alpha)}{(2-\alpha) M(\alpha)} f(t) \\
& +\frac{2 \alpha}{(2-\alpha) M(\alpha)} \int_{0}^{t} f(x) \mathrm{d} x, \quad t \geq 0 .
\end{aligned}
$$

Remark 1. Note that, from the definition in equation (5), the fractional integral of the Caputo-Fabrizio type of function $f$ of order $0<\alpha \leq 1$ is a mean between the function $f$ and its integral of order one, which means

$$
\frac{2(1-\alpha)}{(2-\alpha) M(\alpha)}+\frac{2 \alpha}{(2-\alpha) M(\alpha)}=1,
$$

and therefore,

$$
M(\alpha)=\frac{2}{2-\alpha}, \quad 0<\alpha \leq 1
$$

The advantage of the Caputo-Fabrizio operator over classical Caputo is that there is no singularity for $t=s$.

Definition 3. Due to Caputo and Mauro [19], the Laplace transform for the Caputo-Fabrizio fractional derivative operator of order $0<\alpha \leq 1, M \in \mathbb{N}$, is given by

$$
\begin{aligned}
\mathfrak{Q}\left({ }^{\mathrm{CF}} \mathfrak{D}_{t}^{M+\alpha} f(t)\right)(s) & =\frac{1}{1-\alpha} \mathfrak{L}\left(f^{(M+1)}(t)\right) \mathfrak{L}\left(\exp \left(\frac{-\alpha}{1-\alpha} t\right)\right) \\
& =\frac{s^{M+1} \mathfrak{Q}(f(t))-s^{M} f(0)-S^{M-1} f^{\prime}(0)+\cdots+f^{M}(0)}{s+\alpha(1-s)} .
\end{aligned}
$$

If $M=0$, we get

$$
\mathfrak{Q}\left({ }^{\mathrm{CF}} \mathfrak{D}_{t}^{\alpha} f(t)\right)(s)=\frac{s \mathfrak{Q}(f(t))}{s+\alpha(1-s)} .
$$

The fractional form of equation (3) is given as

$$
{ }^{\mathrm{CF}} \mathfrak{D}_{t}^{\alpha} C+\frac{K K_{L} Q_{0} \rho_{d} C}{R\left(1+K_{L} C\right)}=0 .
$$

\section{Iterative Laplace Transform}

The nonhomogeneous Caputo-Fabrizio fractional differential equation is given as

$$
\begin{gathered}
{ }^{\mathrm{CF}} \mathfrak{D}_{t}^{M+\alpha} f(x, t)=u(x, t)+\phi(f(x, t))+\psi(f(x, t)), \\
M-1<\alpha \leq M, M \in \mathbb{N},
\end{gathered}
$$


with the given condition

$$
\mathfrak{D}_{t}^{K}(x, 0)=\theta_{K}(x), \quad K=0,1,2, \ldots, M-1,
$$

where $u(x, t)$ is a known term, $\phi$ is the linear operator, and $\psi$ is the nonlinear operator.

Applying Laplace transform (8) to both sides of equation (11) yields

$$
\mathfrak{L}(f(x, t))=\lambda(x, s)+\left(\frac{s+\alpha(1-s)}{s^{n+1}}\right) \mathfrak{L}(\phi f(x, t)
$$

$$
+\psi f(x, t))
$$

where

$$
\begin{aligned}
& \lambda(x, s)=\frac{1}{s^{n+1}}\left(s^{n} \theta_{0}(x)+s^{n-1} \theta_{1}(x)+\cdots+\theta_{n}(x)\right)+\frac{s+\alpha(1-s)}{s^{n+1}} \bar{u}(x, s), \\
& f(x, t)=\lambda(x, t)+\mathfrak{Q}^{-1}\left[\left(\frac{s+\alpha(1-s)}{s^{n+1}}\right) \mathfrak{Q}(\phi f(x, t)+\psi f(x, t))\right] .
\end{aligned}
$$

Now, applying the new iterative method [21] yields the solution as an infinite series:

$$
f(x, t)=\sum_{j=0}^{\infty} f_{j}(x, t) .
$$

$$
\phi\left(\sum_{j=0}^{\infty} f_{j}(x, t)\right)=\sum_{j=0}^{\infty} \phi\left(f_{j}(x, t)\right) .
$$

Here, linear function $\phi$ is given as

$$
\begin{aligned}
\psi\left(\sum_{j=0}^{\infty} f_{j}(x, t)\right)= & \psi\left(f_{0}(x, t)\right) \\
& +\sum_{j=1}^{\infty}\left\{\psi\left(\sum_{i=0}^{j} f_{i}(x, t)\right)-\psi\left(\sum_{i=0}^{j-1} f_{i}(x, t)\right)\right\} .
\end{aligned}
$$

In view of equations (15)-(17), equation (14) is equivalent to

$$
\begin{aligned}
\sum_{j=0}^{\infty} f_{j}(x, t)= & \lambda(x, t)+\mathfrak{L}^{-1}\left[\left(\frac{s+\alpha(1-s)}{s^{n+1}}\right) \mathfrak{L}\left(\sum_{j=0}^{\infty} \phi f_{j}(x, t)\right)\right] \\
& +\mathfrak{Q}^{-1}\left[\left(\frac{s+\alpha(1-s)}{s^{n+1}}\right) \mathfrak{L}\left(\psi\left(f_{0}(x, t)\right)+\sum_{j=1}^{\infty}\left\{\psi\left(\sum_{i=0}^{j} f_{i}(x, t)\right)-\psi\left(\sum_{i=0}^{j-1} f_{i}(x, t)\right)\right\}\right)\right] .
\end{aligned}
$$


The recurrence relation is given as

$$
\begin{aligned}
f_{0}(x, t) & =\lambda(x, t), \\
f_{1}(x, t) & =\mathfrak{Q}^{-1}\left[\left(\frac{s+\alpha(1-s)}{s^{n+1}}\right) \mathfrak{Q}\left(\phi\left(f_{0}(x, t)\right)+\psi\left(f_{0}(x, t)\right)\right)\right], \\
f_{p+1}(x, t) & =\mathfrak{Q}^{-1}\left[\left(\frac{s+\alpha(1-s)}{s^{n+1}}\right) \mathfrak{Q}\left(\phi\left(f_{r}(x, t)\right)+\left\{\psi\left(\sum_{i=0}^{p} f_{i}(x, t)\right)-\psi\left(\sum_{i=0}^{p-1} f_{i}(x, t)\right)\right\}\right)\right] .
\end{aligned}
$$

The $p$-term approximate solution is given as

$$
f=f_{0}+f_{1}+f_{2}+\cdots+f_{p-1} .
$$

\section{Results and Discussion}

5.1. Optical Observation. In the beginning, by adding the leaf extract to $1 \mathrm{~mm}$ silver solution, color of the solution turned brown, indicating the immediate and rapid formation of silver nanoparticles. This transformation of color occurs due to the excitation of SPR (surface plasmon resonance) of the silver nanoparticles. Similar results were obtained in an experiment using the root extract of Curculigo orchioides by Dave and Das [6], and the color change observed was brownish yellow to dark brown.

5.2. UV-Vis Spectrophotometer. At the preliminary state, the degradation was identified by color change. The catalytic activity of silver nanoparticles on the degradation of dyes was confirmed using methyl orange as a sample dye. In solar light, silver nanoparticles were used for the degradation of methyl orange, and the amount of dye left was measured at different time intervals. Initially, color of the dye shows deep orange color which changed into light yellow after one hour of incubation along with silver nanoparticles. The absorption spectrum recorded has shown a decrease in the peak at varied time intervals. Calculation shows that the percentage of degradation efficiency of silver nanoparticles was $95.8 \%$ at $70 \mathrm{~h}$ (Table 1). When the exposure time of the dye and silver nanoparticle complex placed in sunlight was increased, the absorption peak also had a decrease. The absorption peak for methyl orange was canter at $660 \mathrm{~nm}$ in the visible region which was reduced, and at last, it disappeared when the reaction time was increased. The whole process was completed after 70 hours of incubation and was recognized by the change of reaction mixture color to colorless (Table 2).

\subsection{Photocatalytic Degradation of Dye}

5.3.1. Visual Observation. Photocatalytic degradation of methyl orange was carried out by using green synthesized silver nanoparticles under solar light. Dye oxidation was initially detected by a shift of hue. Initially, color of the pigment reveals deep orange color modified to light yellow after $1 \mathrm{~h}$ of incubation with silver nanoparticles when exposed to sunlight. Then, the hue changed from bright yellow to pale yellow, and the solution gradually became colorless. Finally, the degradation process was completed at $70 \mathrm{~h}$ and was recognized by the change of reaction mixture color to colorless.

\section{Modelling}

From equation (10), the fractional-order transport equation for the absorption process is given as

$$
{ }^{\mathrm{CF}} \mathfrak{D}_{t}^{\alpha} C+\frac{K K_{L} Q_{0} \rho_{d} C}{R\left(1+K_{L} C\right)}=0,
$$

with $C_{0}=c_{0} e^{-\beta t}, \beta$ is a constant which depends on initial dye concentration.

The second-term approximate solution is given as

$$
\begin{aligned}
C(t)= & c_{0} e^{-\beta t} \\
& -\frac{P c_{0}}{\lambda}\left[(1-\alpha)\left(1+\frac{e^{\beta t}}{\lambda}\right)^{-1}+2 \alpha t+\frac{2 \alpha}{\beta} \log _{e}\left(\frac{\lambda+1}{\lambda+e^{\beta t}}\right)\right],
\end{aligned}
$$

where

$$
\begin{aligned}
\lambda & =c_{0} K_{L}, \\
P & =\frac{K K_{L} Q_{0} \rho_{d}}{R}, \\
\left|\frac{e^{\beta t}}{\lambda}\right| & <1 .
\end{aligned}
$$

Figure 6 gives simulated results of solution (22), with parameters $Q_{0}=10.718, K_{L}=0.308, R^{2}=0.9762, K=0.1$, $R=0.4035 \times 10^{20}, \rho_{d}=0.001$, and $c_{0}=1.7 \times 10^{4}$.

We conclude that concentration decays exponentially with faster rates for initial time and takes long time to reduce for higher initial values. 
TABle 1: Exposure time of the amount of degradation of dye (\%) using silver nanoparticles.

\begin{tabular}{lc}
\hline $\begin{array}{l}\text { Exposure time } \\
\text { (hrs.) }\end{array}$ & $\begin{array}{c}\text { Amount of degradation of dye (\%) using silver } \\
\text { nanoparticles }\end{array}$ \\
\hline 1 & $2.5 \pm 0.15$ \\
2 & $4.7 \pm 0.45$ \\
3 & $7.3 \pm 0.55$ \\
4 & $15.5 \pm 0.47$ \\
10 & $19.5 \pm 0.15$ \\
21 & $25.3 \pm 0.14$ \\
22 & $39.5 \pm 0.65$ \\
24 & $44.2 \pm 0.34$ \\
41 & $47.9 \pm 0.21$ \\
42 & $55.2 \pm 0.22$ \\
44 & $65.2 \pm 0.45$ \\
45 & $75.5 \pm 0.65$ \\
46 & $83.2 \pm 0.37$ \\
48 & $88.9 \pm 0.18$ \\
65 & $89.1 \pm 0.23$ \\
66 & $93.6 \pm 0.88$ \\
70 & $95.8 \pm 0.67$ \\
\hline
\end{tabular}

TABle 2: Exposure time of the amount of degradation of dye (\%) using iron nanoparticles and Fenton-like oxidation.

\begin{tabular}{lc}
\hline $\begin{array}{l}\text { Exposure time } \\
\text { (hrs.) }\end{array}$ & $\begin{array}{c}\text { Amount of degradation of dye (\%) using iron } \\
\text { nanoparticles and Fenton-like oxidation }\end{array}$ \\
\hline 1 & $55 \pm 0.35$ \\
2 & $78 \pm 0.15$ \\
3 & $88.3 \pm 0.25$ \\
4 & $95.2 \pm 0.15$ \\
10 & 99 \\
\hline
\end{tabular}

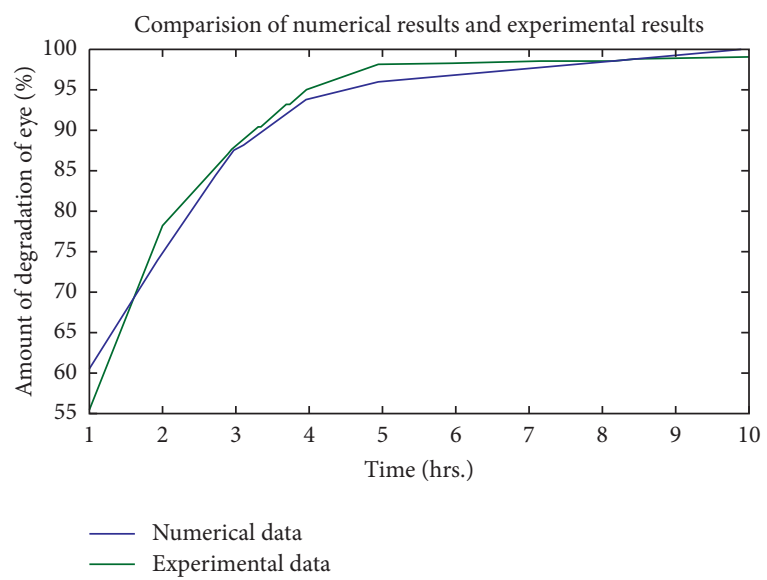

Figure 6: Comparison of numerical results with experimental results using iron nanoparticles and Fenton-like oxidation.

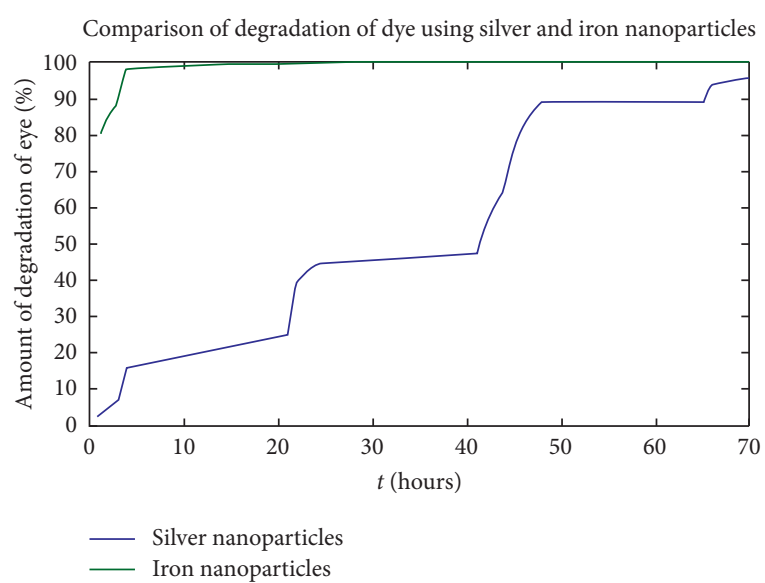

Figure 7: Comparison of the degradation of dye using silver and iron nanoparticles.

\section{Conclusion}

Green synthesis of silver and iron nanoparticles has been carried out using the plant extract of a locally available plant Cordia dichotoma (common name: gonda). Photocatalytic degradation of the dyes using these silver and iron metallic nanoparticles was successfully carried out in the laboratory. The exposure time of the amount of degradation of dye using iron nanoparticles and Fenton-like oxidation was tested, and it was found that it is much lesser with iron nanoparticles compared to the amount of degradation of dye using silver nanoparticles. A numerical fractional model for the transport equation for the concentration process involving Caputo-Fabrizio fractional-order derivatives has been developed to simulate the dye degradation from industry effluents. Iterative Laplace transform method is deployed to solve the model. Model validation has been shown by comparing the analytical simulated solution with experimental results using photocatalytic degradation using silver and iron nanoparticles as eco-friendly and low-cost adsorbents. The simulated results of the model are in good agreement with the experimental results. It is observed that the adsorption process by iron nanoparticles could be well described by the fractional model (Figures 6 and 7). Furthermore, it is clear that the rate of degradation of dye is very sensitive to the initial concentration of dye. From the present study, it is found that iron nanoparticles can be used effectively as low-cost and eco-friendly material for developing large-scale water treatment strategies to remove the toxic dyes in the effluent.

\section{Data Availability}

No data were used to support this study. 


\section{Conflicts of Interest}

The authors declare no conflicts of interest regarding the publication of this article.

\section{Acknowledgments}

This work was supported by the Competitive Research Scheme (CRS) Project funded by TEQIP-III (ATU) Rajasthan Technical University (Grant no. TEQIP-III/ RTU(ATU)/CRS2019-20-02).

\section{References}

[1] D. Acharya, S. Satapathy, D. Tapas Kumar Dora, S. Dave, and J. Das, "Advancement in treatment technologies of biopharmaceutical industrial effluents," Microbial Bioremediation \& Biodegradation, Springer, Singapore, pp. 467-484, 2020.

[2] S. Dave and R. Sharma, "Use of nano-particles in water treatment: a review," International Research Journal of Environmental Sciences, vol. 4, no. 10, pp. 103-106, 2015.

[3] S. Bhakya, S. Muthukrishnan, M. Sukumaran, M. Muthukumar, T. K. Senthil, and M. V. Rao, "Catalytic degradation of organic dyes using synthesized silver nanoparticles: a green approach," Journal of Bioremediation \& Biodegredation, vol. 6, no. 5, 2015.

[4] P. C. Nagajyothi, S. V. Prabhakar Vattikuti, K. C. Devarayapalli, K. Yoo, J. Shim, and T. V. M. Sreekanth, "Green synthesis: photocatalytic degradation of textile dyes using metal and metal oxide nanoparticles-latest trends and advancements," Critical Reviews in Environmental Science and Technology, vol. 50, no. 24, pp. 2617-2723, 2019.

[5] S. D. Purohit, A. M. Khan, D. L. Suthar, and S. Dave, "The impact on raise of environmental pollution and occurrence in biological populations pertaining to incomplete $\mathrm{H}$-function," National Academy Science Letters, vol. 44, 2020.

[6] S. Dave and J. Das, "Technological model on advanced stages of oxidation of wastewater effluent from food industry," in Advanced Oxidation Process for Effluent Treatment Plant, pp. 33-49, Elsevier, Amsterdam, Netherlands, 2020.

[7] S. Dave, "Electrochemical and spectral characterization of silver nano-particles synthesized employing root extract of Curculigo orchioides," Indian Journal of Chemical Technology, vol. 25, no. 2, pp. 201-207, 2018.

[8] L. Mistry, A. M. Khan, and D. L. Suthar, "An epidemic Slia mathematical model with Caputo Fabrizio Fractional derivative," Test Engineering and Management, vol. 83, pp. 26374-26391, 2020.

[9] A. Alaria, A. M. Khan, D. L. Suthar, and D. Kumar, "Application of fractional operators in modelling for charge carrier transport in amorphous semiconductor with multiple trapping," International Journal of Applied and Computational Mathematics, vol. 5, no. 6, pp. 1-10, 2019.

[10] M. Ali Dokuyucu, "Caputo and atangana-baleanu-caputo fractional derivative applied to garden equation," Turkish Journal of Science, vol. 5, no. 1, pp. 1-7, 2020.

[11] M. Ali Dokuyucu and E. Celik, "Analyzing a novel coronavirus model (COVID-19) in the sense of caputo-fabrizio fractional operator," Applied and Computational Mathematics an International Journal, vol. 20, no. 1, pp. 49-69, 2021.

[12] M. Ali Dokuyucu, E. Celik, H. Bulut, and H. Mehmet Baskonus, "Cancer treatment model with the Caputo-Fabrizio fractional derivative," The European Physical Journal Plus, vol. 133, no. 3, p. 92, 2018.

[13] S. I. Butt, M. Nadeem, and G. Farid, "On Caputo fractional derivatives via exponential s-convex functions," Turkish Journal of Science, vol. 5, no. 2, pp. 140-146, 2020.

[14] I. Koca, E. Akcetin, and P. Yaprakdal, "Numerical approximation for the spread of SIQR model with Caputo fractional order derivative," Turkish Journal of Science, vol. 5, no. 2, pp. 124-139, 2020.

[15] J. Zhao, S. I. Butt, J. Nasir, Z. Wang, and I. Tlili, "Hermite-jensen-mercer type inequalities for Caputo fractional derivatives," Journal of Function Spaces, vol. 2020, Article ID 7061549, 11 pages, 2020.

[16] S. Zhao, S. I. Butt, W. Nazeer, J. Nasir, M. Umar, and Y. Liu, "Some Hermite-Jensen-Mercer type inequalities for k-Caputo-fractional derivatives and related results," Advances in Difference Equations, vol. 2020, no. 1, p. 262, 2020.

[17] F. Doulati Ardejani, K. Badii, N. Yousefi Limaee et al., "Numerical modelling and laboratory studies on the removal of Direct Red 23 and Direct Red 80 dyes from textile effluents using orange peel, a low-cost adsorbent," Dyes and Pigments, vol. 73, no. 2, pp. 178-185, 2007.

[18] E. Quiroga, P. M. Centres, N. A. Ochoa, and A. J. RamirezPastor, "Fractional statistical theory of adsorption applied to protein adsorption," Journal of Colloid and Interface Science, vol. 390, no. 1, pp. 183-188, 2013.

[19] M. Caputo and F. Mauro, "A new definition of fractional derivative without singular kernel," Progress in Fractional Differentiation and Applications, vol. 1, pp. 1-13, 2015.

[20] J. Losada and J. Nieto, "Properties of a new fractional derivative without singular kernel," Progress in Fractional Differentiation and Applications, vol. 1, pp. 87-92, 2015.

[21] A. Shaikh, A. Tassaddiq, K. S. Nisar, and D. Baleanu, "Analysis of differential equations involving Caputo-Fabrizio fractional operator and its applications to reaction diffusion equations," Advances in Difference Equations, vol. 178, pp. 1-14, 2019. 